\title{
La incidencia de la Planificación estratégica en el desempeño del Marketing: Una revisión crítica
}

\section{The Impact of Strategic Planning on Marketing Performance: a critical review}

\author{
José G. Ruiz-Andrade* \\ Universidad Autónoma de Baja California - México \\ ORCID iD:https://orcid.org/0000-0001-7019-112X \\ gabruiz@uabc.edu.mx

\section{Claudia L. Solís-Palafox} \\ Universidad Autónoma de Baja California - México \\ ORCID iD: https://orcid.org/0000-0002-8770-7791 \\ claudia.solis@uabc.edu.mx
}

\section{Norma A. López-Luna}

Universidad Autónoma de Baja California - México

ORCID iD: https://orcid.org/0000-0003-4546-9956

lopez.norma@uabc.edu.mx

* Autor a quien debe ser dirigida la correspondencia
Fecha de recepción: 15/09/2021

Fecha de evaluación: 01/10/2021

Fecha de aceptación: 30/10/2021

Cómo citar: Ruiz-Andrade, J., Solís-Palafox, C., \& López-Lu$n a, N$. (2021). La incidencia de la Planificación estratégica en el desempeño del Marketing: Una revisión crítica. Revista Científica Anfibios, 4(2), 36-44. https://doi.org/10.37979/afb.2021v4n2.93

\section{Resumen}

El entorno cambiante en el que están inmersas las organizaciones en la actualidad, lleva a que estás tomen decisiones estratégicas bien fundamentadas, es por esto que los gerentes de marketing necesitan practicar de manera constante la formalización, implementación y evaluación de los planes estratégicos de marketing. La literatura previa sustenta la importancia de esta planificación en los resultados organizacionales, sin embargo, existen algunas barreras que tienen que ver con la cultura y las políticas, por ejemplo, la confianza en la planificación y sus resultados, la resistencia al cambio y la cognitiva que tienen que ver con la falta de conocimiento, habilidades e innovación. Es por esto que se planea un planteamiento crítico de la incidencia de la Planificación estratégica en el desempeño del marketing a través de una revisión de la literatura. Los resultados apuntan a que las relaciones entre la planeación estratégica del marketing y el impacto que genera dentro de las empresas al llevarlo a la práctica tiene resultados positivos en el desempeño, no obstante, se requiere de alto conocimiento del entorno para que la planificación sea efectiva respecto a los resultados.

Palabras clave

Planificación estratégica; desempeño de marketing; estrategia; cliente

\begin{abstract}
The changing environment in which organizations are immersed today, leads them to make informed strategic decisions, which is why marketing managers need to constantly practice the formalization, implementation and evaluation of strategic marketing plans. Previous literature supports the importance of this planning in the results of organizations, however there are some barriers that have to do with culture and policies, for example, confidence in planning and its results, resistance to change and cognitive barriers that have to do with lack of knowledge, skills and innovation. This is why a critical approach to the incidence of strategic planning on marketing performance is planned through a literature review. The results point out that the relationship between strategic marketing planning and the impact it generates within companies when put into practice has positive results in performance; however, a high level of knowledge of the environment is required for planning to be effective with respect to results.
\end{abstract}




\section{Keywords}

Strategic planning; marketing performance; strategy; customer

\section{Introducción}

En un mundo actual, tan globalizado, diariamente los clientes se atiborran de información a cada minuto, en cualquier lugar y a cada instante por medio de campañas publicitarias (K. H. Kim et al., 2008; Morgan \& Rego, 2009; Romaniuk \& Wight, 2009; Rossiter \& Larry, 1987), pero, como ya bien lo comenta en una de sus frases célebres, uno de los pioneros de la mercadotecnia, John Wanamaker: "La mitad del dinero que gasto en publicidad se desperdicia, el problema es que no se cual mitad es." En este punto, el marketing tiene diversas definiciones, por ejemplo, la revista líder especializada en mercadotecnia, publicidad y medios a nivel nacional dentro de México, cita al considerando, padre de la mercadotecnia moderna, Philip Kotler, donde define la mercadotecnia como, esta disciplina es un proceso social y administrativo mediante el cual grupos e individuos obtienen lo que necesitan y desean a través de generar, ofrecer e intercambiar productos de valor con sus semejantes (P. Kotler, 1984; P Kotler, 1998)

El problema, no son los productos y servicios que se ofertan por parte de las empresas, que están diseñados para satisfacer y resolver las necesidades del cliente, sino, que no todos los clientes comparten las mismas necesidades y problemáticas(Echchakoui, 2017; Y. K. Kim \& Sullivan, 2019; Peesker et al., 2019; Spiteri \& Dion, 2004). Por lo tanto, la nula o carente implementación de mercadotecnia estratégica en los planes de trabajo resultan una verdadera problemática para aquellas empresas empíricas que entre comillas, "creen" que con solo anunciar, publicar o difundir su bien o servicio por canales de difusión tradicionales o de redes sociales tendrán éxito (Esquivel, 2019; Kumar \& RRK, 2017; Larke et al., 2018; R. R. Mullins \& Panagopoulos, 2019).

Es por esto que se propone analizar la incidencia de la planificación estratégica en el desempeño del marketing a través de una revisión de la literatura, es decir, analizar las publicaciones más relevantes que vinculen estas áreas de conocimiento y permita dilucidar esta relación que parece tan evidente pero que es mínimamente implementada en los entornos organizaciones más reducidos. En ese sentido, el valor agregado de la presente propuesta está centrada en presentar una crítica respecto a la falta de implementación de la planificación estratégica y como esta puede incidir en el desempeño del marketing, es así, que se pretende demostrar que la importancia de planear estratégicamente las campañas de marketing y a partir de allí comenzar a generar desempeños óptimos para las organizaciones.

Tomando en cuenta lo anterior, se plantean unos referentes teóricos conceptualizando temáticas centrales como el marketing o mercadotecnia y la planificación estratégica con la finalidad de obtener puntos de vista que vinculen ambas temáticas. Seguidamente se presenta la metodología anunciando una revisión de la literatura basada en publicaciones científicas con alto grado de citación e impacto en la comunidad académica, adicionalmente, también se presenta la lectura crítica a las que serán examinadas estos artículos y finalmente se plantearan los resultados encontrando la importancia de la estratégica y su planificación en los resultados del marketing llamando la atención de gerentes de marketing para que en la práctica implementen en mayor medida estas recomendaciones.

\section{Referentes Teóricos}

De acuerdo con McDonald (1996) las empresas que cuentan con sistemas de planificación de marketing bien establecido y acorde con sus clientes serán más exitosas que otras empresas que compitan en el mismo ambiente. En este punto se define la mercadotecnia en la actualidad como el conjunto de técnicas y habilidades que tienen como objetivo mejorar la comercialización de un producto (Hanggraeni et al., 2019; Matthyssens, 2019; R. R. Mullins \& Panagopoulos, 2019; Sainidis et al., 2019). Aunque esta definición podría ser ambigua, en el presente artículo se hace referencia a la importancia de llevar a cabo sistemas de marketing que se puedan medir, en este contexto Harris (1996) afirma que la planificación debe ser específica y derribar la barrera cultural con la investigación en el tema.

Identificar las ventajas competitivas de la organización permite una mejor planificación estratégica de marketing y rendimiento (Asare et 
al., 2015; Grinstein, 2008; Magnotta \& Johnson, 2020), sin embargo, esto suele ser complicado por tres razones: la primera porque no se identifican las capacidades intrínsecas de la organización, segundo porque para desarrollar una capacidad de marketing se debe poner en práctica los conocimientos y habilidades intangibles para transformar los insumos de marketing en productos y el tercero de debe a las turbulencias ambientales (Day, 1994; Grant, 1996; Vorchres, 1998; Katsikeas et al., 2016; Srinivasan \& Lohith, 2017).

Cuando se realizaban los primeros estudios de planificación estratégica de marketing muchos gerentes la percibían como algo innecesario (Piercy y Morgan, 1994), sin embargo, los esfuerzos de muchos investigadores demuestran lo contrario (Agostini et al., 2015; Dalecki, 2016; Milichovsky \& Simberova, 2015).

Con el nombre de Teoría de segmentación de mercado de trabajo, así lo menciona Fernández (2009), se suele englobar un conjunto de enfoques, bastante diverso en cuanto a sus orígenes y contenidos, que comenzaron a surgir a finales de los años sesenta impulsados por el descontento hacia la explicación neoclásica del mercado de trabajo. Por esta misma razón, la segmentación de mercado juega un papel vital al momento previo de ofertar y diseñar un bien o servicio que resuelva las necesidades específicas de un cliente potencial (Aragón Sánchez \& Bañón Rubio, 2006; Keller, 2003; Teece, 2007).

Diversos estudios han demostrado la capacidad de la teoría de segmentación de mercado, la cual, es un vivo ejemplo de saber primero hacia qué tipo de mercado va dirigido los productos o servicios, ya que es una parte fundamental para tener éxito en ello, no porque el producto no sea bueno, simplemente son factores externos que ya están comprobados que influyen (Cleeren et al., 2010; Halim et al., 2019; Lupoli Junior \& Dos Santos, 2019; Morgan \& Rego, 2009) .

Para Kotler \& Keller, (2006) los economistas describen el mercado como el grupo de compradores y vendedores que realizan transacciones sobre un producto o clases de productos, siempre y cuando se tenga el conocimiento previo a que mercado va dirigido. En ese sentido, para poder desarrollar un producto dentro de la mente del consumidor a nivel neuronal, se tienen que tomar en cuenta aspectos con base a los hábitos, deseos y necesidades del mercado objetivo. Esta psicología nos lleva a emplear las bases de las $4 p$, concepto que, desarrollado en 1950 por Neil Borden, el cual propuso 12 elementos que conformaban las responsabilidades de un Director de Marketing, el cual, diez años más tarde, en 1960 el profesor Jerome McCarthy simplificaría a tan solo cuatro elementos: Producto, Precio, Plaza y Promoción.

Jerome McCarthy después de haber reducido a tan solo cuatro elementos el concepto de Marketing mix, ofrece una lección que para poder llegar a la mente del consumidor es necesario anticiparse a los requerimientos y necesidades del consumidor potencial.

Otro concepto asociado a la teoría del ciclo de vida del producto, una teoría económica desarrollada por Raymond Vernon en 1966, la cual sugiere que todo producto tiene una etapa de vida útil, pasando por varias etapas desde el inicio, crecimiento, madurez hasta llegar a su declive. La mercadotecnia es parte fundamental de toda empresa, pues como mencionaba antes, no solo es "anunciar" como la mayoría de la gente tiene pensado, es desde un inicio como lo menciona Vernon, al inicio del producto o servicio, la empresa debe despertar el interés del cliente potencial para su consumo, una vez cautivada la atención, empeñar sus esfuerzos por cautivar al mercado fiel y ampliar su mercado a más consumidores potenciales con perfil afín, a medida que va creciendo y madurando, hacer notar la diferencia de la misma competencia, para mantener el mayor tiempo posible en ese punto de la curva antes del declive.

Respecto a la planificación estratégica, de acuerdo con Barbosa et al., (2020) en un mercado cada vez más competitivo, bajo nuevas perspectivas y paradigmas, por ejemplo, el lucro no es el único objetivo, o en la actualidad, el lucro debe ser el resultado de hacer buenas obras; la organización tiene beneficios compartidos con la sociedad; por la necesidad de proteger recursos y garantizar que la disponibilidad futura y el crecimiento económico sean limitados. Es por esto que los gerentes deben aceptar el desafío de aplicar el pensamiento y las habilidades estratégicas (razón ética, sentido de responsabilidad, espíritu emprendedor, conciencia) de líderes efectivos, es decir, deben ir más allá de planificar, organizar, ejecutar y controlar las actividades comerciales efectivas. 
La gestión estratégica requiere que los gerentes tengan la capacidad de supervisar e interpretar la realidad del entorno organizacional interno y externo, y utilizarlo para el desarrollo de la situación estratégica de la empresa, y transformarlo a través de la declaración de valores, visión, misión y metas estratégicas (Baykasollu \& Gölcük, 2015; Dororthy Leonard-Barton, 1992; Matthyssens, 2019). A partir de ahí, puede definir una estrategia comercial global y luego proponer metas y acciones específicas. Por tanto, no comprende la importancia de los ajustes estratégicos en todo el proceso (Garcia et al., 2013; Jeong \& Harrison, 2017).

\section{Metodología}

Enmarcado en un tipo de investigación cualitativa y en específico de corte critico-analítico (Schwalb Helguero \& García-Arrizabalaga, 2019), se busca analizar la incidencia de la planificación estratégica en el desempeño del Marketing por medio de una revisión de la literatura, para tales fines, se planteó una búsqueda y recopilación de artículos científicos tomando como referencia palabras claves como "gestión estratégica", "planificación estratégica" y "desempeño de marketing" tanto en idioma inglés como español vinculadas al tema de investigación propuesto en la base de datos científicas de Scopus y Web Of science (WOS). Los criterios de inclusión obedecen a los artículos citados mayormente y que tengan relación directa con la temática planteada. Adicionalmente, se plantean analizar estas publicaciones bajo una revisión crítica (Pinillos et al., 2018), con la finalidad de enfatizar la importancia que tiene la incidencia de la planificación estratégica en el desempeño del Marketing llamando la atención de los gerentes en la práctica, de tal manera que esta relación se posicione más como un elemento empírico que como uno teórico.

\section{Resultados}

Para poder llevar a cabo un marketing estratégico dentro de una empresa, es primordialmente necesario que, la organización invierta en un grupo o equipo de trabajo dentro de la empresa que se dedique a comunicar de manera efectiva las características y beneficios que ofrecen(Andotra \& Gupta, 2016; Keller, 2010; Magnotta \& Johnson, 2020; Sainidis et al., 2019). Una muestra de este pensamiento corresponde al los resultados empíricos desarrollados por Phillips et al., (2001) en este estudio realizado a ocho hoteles dentro de Reino Unido, se pudo dar cuenta que la aplicación y uso de mercadotecnia a nivel empírico da resultados muy bajos o casi nulos.

Esto requiere que el equipo de marketing de la empresa realice planes estratégicos que definan la forma en que competirán con el demás mercado y la filosofía de trabajo que proyecto tanto al interior de la empresa como al exterior (Kaski et al., 2018; Larke et al., 2018; R. Mullins et al., 2019).

No cabe duda que la mercadotécnica es posible de encontrar en todo momento en la vida del consumidor, pero si hablamos de poder transmitirlo como empresa, en necesario definitivamente contar de manera efectiva con un equipo y plan de mercadotecnia estratégica (Cuamea Velázquez et al., 2019).

Por consiguiente, Phillips et al., (2001) plantearon un estudio de 50 principales grupos hoteleros de Reino Unido en cual se analizaron diferentes variables para llegar a la pregunta de investigación ¿Cuáles son los efectos interactivos de la planificación estratégica y desempeño con énfasis en identificar la contribución de los insumos variables al rendimiento?

Estas variables miden tanto el rendimiento de marketing en áreas de formalidad, participación de mercado, sofisticación, eficiencia, eficacia, adaptabilidad, posteriormente se utilizó la escala de Likert para medir los resultados y un sistema que media la correlación de unas variables con otras, los resultados son los siguientes.

Se anticipa que los resultados confirmarán que una mejor coincidencia entre la planificación estratégica en los procesos y el entorno externo resultarán en un aumento desempeño organizacional.

El desempeño de las instituciones fue determinado por indicadores como; Eficacia: Porcentaje de ocupación, tarifa media de la habitación, y crecimiento de ventas por habitación (Medeiros et al., 2019). Eficiencia: retorno de la inversión, margen de beneficio (Hernández, 2001); Adaptabilidad: Numero de servicios / productos exitosos introducidos porcentaje de ventas contabilizadas por nuevos servicios / productos, ente otros.

Phillips et al., (2001) calcularon el alfa de Cronbach de cada factor y se encontró que el nivel de participación en el proceso de planificación, minuciosidad de la planificación y formalidad de la pla- 
nificación, respectivamente, tienen un alto nivel de coherencia entre sí, mientras que la sofisticación de la planificación no siempre esta liada a las otras tres.

Las variables que tuvieron un alto nivel de participación fueron; planificación, sofisticación en la planificación que destaca el uso de la sabiduría empresarial en la planificación empresarial.

Otro factor que tiene alto nivel es que hace referencia a la frecuente comunicación entre los miembros del departamento de marketing de la oficina central. Otra de las variables altamente cargadas son las relativas a la competencia, la percepción de muchas "guerras promociones en el sector hotelero son altas.

Existe otra variable significativa que se basa en la diferenciación de productos y la del factor 6 que hace énfasis al marketing y las variables son la percepción de que los especialistas de marketing ejercen más influencia que los contadores en el proceso de planificación a largo plazo.

Una de las variables que salió negativa es la relacionada con la búsqueda de una estrategia enfocada, mientras que la estrategia de bajo precio salió positiva. Este factor se interpreta como "cultura de precios bajos, que tiene que ver con bajar los precios en el sector hotelero sin haber segmentado previamente a su mercado.

Otro hito importante en cuanto la relación entre la planificación estratégica en el desempeño del Marketing Boone \& Roehm, (2002) sostienen que la "idoneidad" de una solución de posicionamiento específica es una consideración clave en toda investigación de marketing. "Apropiada" significa que no solo se ha determinado la solución de posicionamiento óptima, sino que también se ha especificado correctamente el número apropiado de segmentos de mercado.

En ese sentido, los autores manifiestan que la determinación del número adecuado de segmentación es fundamental tanto para la planificación estratégica como para el rendimiento de marketing, es por es por eso que $\mathrm{Al}$ especificar detalladamente el número apropiado de segmentos de mercado, los especialistas en marketing pueden segmentar en exceso el mercado y tratar segmentos de audiencia individuales que se pueden abordar de manera inclusiva. Por el contrario, si el número correcto de segmentos de mercado es insuficiente, es posible que los especialistas en marketing no puedan identificar segmentos de mercado diferentes y factibles para ubicar individualmente la segmentación del mercado.

Es así que Boone \& Roehm, (2002) El problema de la segmentación insuficiente y excesiva del mercado puede ser resuelto por los Criterios de Membresía (MCC),). Utilizando conjuntos de datos del mundo real y artificiales, estos autores plantearon pruebas empíricas, lo comparamos con métodos existentes para determinar el número de segmentos de mercado y demostramos sus ventajas al evaluar la aplicabilidad de diferentes segmentos de mercado.

Es así que se planteó la incidencia de la Planificación estratégica en el desempeño del Marketing, extendiendo los argumentos respecto a la partición de ambos elementos y a partir de esta se proponen unas conclusiones, discusiones y críticas.

\section{Conclusiones}

El estudio proporciona un análisis multidimensional de la planificación estratégica de marketing y relación de desempeño. Los hallazgos sugieren que la cuestión no es si la planificación estratégica de marketing afecta el rendimiento, sino más bien que técnicas del marketing se requieren para mejorar el rendimiento.

Las capacidades esenciales de marketing deben determinarse evaluando las habilidades y competencias de manera individual, grupas y organizacional.

Los hallazgos de esta investigación sugieren que el desafío para las organizaciones es enfatizar una planeación estratégica formal, sofisticada y comunicación efectivas, junto con una estrategia de diferenciación.

Es así que se considera que, aun cuando en un principio existió cierta resistencia al cambio a la planificación estratégica por parte de varios gerentes de marketing, al día de hoy hay un avance significativo en las organizaciones, aunque muchas siguen sin utilizar estas técnicas y capacidades, varios hallazgos de investigación respaldan la importancia de invertir conocimiento y dinero en esta área. 


\section{Referencias}

Agostini, L., Filippini, R., \& Nosella, A. (2015). Brand-Building Efforts and Their Association with SME Sales Performance. Journal of Small Business Management, 53(S1), 161-173. https://doi. org/10.1111/jsbm.12185

Andotra, N., \& Gupta, R. (2016). Impact of environmental turbulence on market orientation-business performance relationship in SSIs. Global Business Review, 17(4), 806-820. https://doi. org/10.1177/0972150916645679

Aragón Sánchez, A., \& Bañón Rubio, A. (2006). Factores explicativos del éxito competitivo: el caso de las PyMEs del estado de Veracruz. Contaduría y Administración, 216(June), 35-69.

Asare, R., Akuffobea, M., Quaye, W., \& Atta-Antwi, K. (2015). Characteristics of micro, small and medium enterprises in Ghana: Gender and implications for economic growth. African Journal of Science, Technology, Innovation and Development, 7(1), 26-35. https://doi.org/10.1080/20421338.2014.9 79651

Barbosa, M., Castañeda -Ayarza, J. A., \& Lombardo Ferreira, D. H. (2020). Sustainable Strategic Management (GES): Sustainability in small business. Journal of Cleaner Production, 258. https://doi. org/10.1016/j.jclepro.2020.120880

Baykasołlu, A., \& Gölcük, I. (2015). Development of a novel multiple-attribute decision making model via fuzzy cognitive maps and hierarchical fuzzy TOPSIS. Information Sciences, 301, 75-98. https:// doi.org/10.1016/j.ins.2014.12.048

Boone, D. S., \& Roehm, M. (2002). Evaluating the Appropriateness of Market Segmentation Solutions Using Artificial Neural Networks and the Membership Clustering Criterion. Marketing Letters, 13(4), 317-333. https://doi.org/10.1023/A:1020321132568

Cleeren, K., Verboven, F., Dekimpe, M. G., \& Gielens, K. (2010). Intra- and interformat competition among discounters and supermarkets. Marketing Science, 29(3), 456-473. https://doi.org/10.1287/ mksc. 1090.0529

Cuamea Velázquez, O., Ramos Higuera, K. y, \& Galván León, J. A. (2019). Efecto De La Mercadotecnia Digital En La Elección De Mipymes Vinícolas En El Valle De Guadalupe, México. Empresa. Investigación y Pensamiento Crítico, 8(3), 150-177. https://doi.org/http://dx.doi.org/10.17993/3c emp.2019.080339.150-177

Dalecki, L. (2016). Sales as entrepreneurship at Ewing Kauffman's Marion Laboratories. Journal of Research in Marketing and Entrepreneurship, 18(1), 14-26. https://doi.org/10.1108/jrme-02-20150009

Dororthy Leonard-Barton. (1992). Core Capabilities and Core Rigidities: A Paradox in Managing New Product Development. Strategic Management Journal, 13(Strategy Process: Managing Corporate Self-Renewal), 111-125. https://doi.org/10.1017/CBO9781107415324.004

Echchakoui, S. (2017). Effect of salesperson personality on sales performance from the customer's perspective: Application of socioanalytic theory. European Journal of Marketing, 51(9-10), 17391767. https://doi.org/10.1108/EJM-03-2016-0147

Esquivel, I. V. (2019). Factores Críticos De Éxito En El Comercio Digital De Las Pymes Exportadoras Costarricenses. Tec Empresarial, Enero-Abril, 13(1), 19-34. https://doi.org/10.18845/te.v13i1.4293

Fernández, E. (2010). La teoría de la segmentación del mercado de trabajo: enfoques, situación actual y perspectivas de futuro. Investigación económica, vol. LXIX, 273, julio-septiembre de 2010, pp. 115-150. http://www.scielo.org.mx/pdf/ineco/v69n273/v69n273a4.pdf 
Garcia, F., Jin, B. C., \& Salomon, R. (2013). Does inward foreign direct investment improve the innovative performance of local firms? Research Policy, 42(1), 231-244. https://doi.org/10.1016/j. respol.2012.06.005

Grinstein, A. (2008). The relationships between market orientation and alternative strategic orientations. 42(1), 115-134. https://doi.org/10.1108/03090560810840934

Halim, K. K., Halim, S., \& Felecia. (2019). Business intelligence for designing restaurant marketing strategy: A case study. Procedia Computer Science, 161, 615-622. https://doi.org/10.1016/j. procs.2019.11.164

Hanggraeni, D., Ślusarczyk, B., Sulung, L. A. K., \& Subroto, A. (2019). The Impact of Internal, External and Enterprise Risk Management on the Performance of Micro, Small and Medium Enterprises. Sustainability, 11(7), 2172. https://doi.org/10.3390/su11072172

Hernández, R. (2001). Elementos de Competitividad Sistemica de las PYMEs del Istmo Centroamericano. In Naciones Unidas - CEPAL: Vol. MEX (Issue L.499).

McDonald, M. (1996). Strategic marketing planning: theory, practice and research agendas. Journal of Marketing Management, 12(1-3), 4-27. https://doi.org/10.1080/0267257X.1996.9964398

Jeong, S. H., \& Harrison, D. A. (2017). Glass breaking, strategy making, and value creating: Meta-analytic outcomes of women as ceos and tmt members. Academy of Management Journal, 60(4), 1219-1252. https://doi.org/10.5465/amj.2014.0716

John Wanamaker Collection, 1827-1987. https://hsp.org/sites/default/files/legacy_files/migrated/ findingaid2188wanamaker.pdf

Kaski, T., Niemi, J., \& Pullins, E. (2018). Rapport building in authentic B2B sales interaction. Industrial Marketing Management, 69(August), 235-252. https://doi.org/10.1016/j.indmarman.2017.08.019

Katsikeas, C. S., Morgan, N. A., Leonidou, L. C., \& Hult, G. T. M. (2016). Assessing performance outcomes in marketing. Journal of Marketing, 80(2), 1-20. https://doi.org/10.1509/jm.15.0287

Keller, K. L. (2003). Brand Synthesis: The Multidimensionality of Brand Knowledge. Journal of Consumer Research, 29(4), 595-600. https://doi.org/10.1086/346254

Keller, K. L. (2010). Brand equity management in a multichannel, multimedia retail environment. Journal of Interactive Marketing, 24(2), 58-70. https://doi.org/10.1016/j.intmar.2010.03.001

Kim, K. H., Kim, K. S., Kim, D. Y., Kim, J. H., \& Kang, S. H. (2008). Brand equity in hospital marketing. Journal of Business Research, 61(1), 75-82. https://doi.org/10.1016/j.jbusres.2006.05.010

Kim, Y. K., \& Sullivan, P. (2019). Emotional branding speaks to consumers' heart: the case of fashion brands. Fashion and Textiles, 6(1). https://doi.org/10.1186/s40691-018-0164-y

Kotler, P. (1984). Marketing management: analysis, planning, implementation and control. (E. Cliffs (ed.); 6th ed.). Prentice-Hall.

Kotler, P. (1998). Marketing Management: Analysis, Planning and Control. (Englewood Cliffs (ed.); Prentice-H).

Kotler, P., y Keller, K., (2016). Dirección de Marketing. Pearson Educación. Decimoquinta Edición. https://docs.google.com/r?a=v\&pid=sites\&srcid=ZGVmYXVsdGRvbWFpbnxhZ3NwZXJZb25hbGV8Z3g6NDM3MGQxZGE5YzFhNDcwZA

Kotler, Philip, \& Keller, K. (2006). Marketing Management. Upper Saddle River. 
Kumar, V., \& RRK, S. (2017). An empirical investigation of critical success factors influencing the successful TQM implementation for firms with different strategic orientation. International Journal of Quality \& Reliability Management, 34(9), 1-5.

Larke, R., Kilgour, M., \& O’Connor, H. (2018). Build touchpoints and they will come: transitioning to omnichannel retailing. International Journal of Physical Distribution and Logistics Management, 48(4), 465-483. https://doi.org/10.1108/IJPDLM-09-2016-0276

Lupoli Junior, J. G., \& Dos Santos, Y. M. (2019). Abordagens de marketing e orientação para mercado aplicadas às médias empresas brasileiras. Revista Ciências Administrativas, 25(3), 1-17. https://doi.org/10.5020/2318-0722.2019.8296

Magnotta, S. R., \& Johnson, C. M. (2020). The role of sales team intragroup conflict on critical job outcomes. Industrial Marketing Management, 84(March), 126-137. https://doi.org/10.1016/j. indmarman.2019.06.003

Matich, D. (2001). Redes neuronales: Conceptos básicos y aplicaciones. Universidad Tecnológica Nacional - Facultad regional Rosario. Departamento de Ingeniería Química. https://www. frro.utn.edu.ar/repositorio/catedras/quimica/5_anio/orientadora1/monograias/matich-redesneuronales.pdf

Matthyssens, P. (2019). Reconceptualizing value innovation for Industry 4.0 and the Industrial Internet of Things. Journal of Business and Industrial Marketing, 34(6), 1203-1209. https://doi. org/10.1108/JBIM-11-2018-0348

Medeiros, V., Godoi, L. G., \& Teixeira, E. C. (2019). La competitividad y sus factores determinantes: Un análisis sistémico para países en desarrollo. Cepal Review, 2019(129), 7-27. https://doi. org/10.18356/9c2a7060-es

Merca 2.0. (2010). Phillip Kotler y su definición de Mercadotecnia. Revista Digital. https://www. merca20.com/philip-kotler-da-su-definicion-de-mercadotecnia/

Milichovsky, F., \& Simberova, I. (2015). Marketing Effectiveness: Metrics for Effective Strategic Marketing.: University of Warwick eResources. 26(2), 211-219. http://0-eds.a.ebscohost.com. pugwash.lib.warwick.ac.uk/eds/pdfviewer/pdfviewer?sid=5fc4b05e-5741-4430-9f05-6b4d$294 \mathrm{f0eb} 8 \% 40$ sessionmgr4008\&vid=1\&hid=4113

Morgan, N. A., \& Rego, L. L. (2009). Brand portfolio strategy and firm performance. Journal of Marketing, 73(1), 59-74. https://doi.org/10.1509/jmkg.73.1.59

Mullins, R. R., \& Panagopoulos, N. G. (2019). Understanding the theory and practice of team selling: An introduction to the special section and recommendations on advancing sales team research. Industrial Marketing Management, 77(xxxx), 1-3. https://doi.org/10.1016/j.indmarman.2018.03.001

Mullins, R., Menguc, B., \& Panagopoulos, N. G. (2019). Antecedents and performance outcomes of value-based selling in sales teams: a multilevel, systems theory of motivation perspective. Journal of the Academy of Marketing Science. https://doi.org/10.1007/s11747-019-00705-2

Paul A. Phillips, Fiona M. Davies \& Luiz Moutinho (2001) The Interactive Effects of Strategic Marketing Planning and Performance: A Neural Network Analysis, Journal of Marketing Management, 17: 1-2, 159-182, DOI: 10.1362 / 0267257012571438

Peesker, K. M., Ryals, L. J., Rich, G. A., \& Boehnke, S. E. (2019). A qualitative study of leader behaviors perceived to enable salesperson performance. Journal of Personal Selling and Sales Management, 39(4), 319-333. https://doi.org/10.1080/08853134.2019.1596816 
Phillips, P. A., Davies, F. M., \& Moutinho, L. (2001). The Interactive Effects of Strategic Marketing Planning and Performance: A Neural Network Analysis. Journal of Marketing Management, 17(1-2), 159-182. https://doi.org/10.1362/0267257012571438

Piercy, N. F., \& Morgan, N. A. (1994). The marketing planning process: behavioral problems compared to analytical techniques in explaining marketing plan credibility. Journal of Business Research, 29(3), 167-178.

Pinillos, A., Fernández Fernandez, J. L., \& Fernández Mateo, J. (2018). Revisión crítica de la dimensión Gobierno Corporativo en los cuestionarios de los Índices de Sostenibilidad A critical review of the Corporate Governance dimension in the Sustainability Indexes questionnaires. In Revista de Comunicación 17 (2), 2018 (Vol. 17, Issue 2).

Romaniuk, J., \& Wight, S. (2009). The Influences of Brand Usage on Response to Advertising Awareness Measures. International Journal of Market Research, 51(2), 203-218. https://doi. org/10.2501/s1470785309200426

Rossiter, J. R., \& Larry, P. (1987). Advertising and Promotion Management. McGraw-Hill Book Company.

Sainidis, E., Robson, A., \& Heron, G. (2019). Environmental turbulence and the role of business functions in the manufacturing strategy debate: The case of UK-based SMEs and the Great Recession. Journal of General Management, 44(4), 190-208. https://doi.org/10.1177/0306307019832498

Schwalb Helguero, M. M., \& García-Arrizabalaga, I. (2019). A scale to measure the social responsibility of marketing. Revista Venezolana de Gerencia, 24(87).

Spiteri, J. M., \& Dion, P. A. (2004). Customer value, overall satisfaction, end-user loyalty, and market performance in detail intensive industries. Industrial Marketing Management, 33(8), 675-687. https://doi.org/10.1016/j.indmarman.2004.03.005

Srinivasan, R., \& Lohith, C. P. (2017). Strategic Marketing and Innovation for Indian MSMEs. Strategic Marketing and Innovation for Indian MSMEs, 31-39. https://doi.org/10.1007/978-98110-3590-6

Teece, D. J. (2007). Explicating dynamic capabilities: The nature and microfoundations of (sustainable) enterprise performance. Strategic Management Journal, 18(1), 1319 - 1350. https://doi. org/10.1002/smj

Vernon, R. (1966). Teoría del ciclo de vida de producto. Recuperado de documento realizado por Ramos, V. (2019) https://idoc.pub/documents/idocpub-mwl1vxqj39lj

Wanamaker, J. (2018). The historical society of Pennsylvania, collection 2188 